\title{
Publisher Correction: Spontaneous regression of micro-metastases following primary tumor excision: a critical role for primary tumor secretome
}

Lee Shaashua $^{1 \dagger}$, Anabel Eckerling $^{1 \dagger}$, Boaz Israeli ${ }^{1}$, Gali Yanovich², Ella Rosenne', Suzana Fichman-Horn³, Ido Ben Zvi ${ }^{4}$, Liat Sorski ${ }^{1}$, Rita Haldar ${ }^{1}$, Ronit Satchi-Fainaro ${ }^{5}$, Tamar Geiger ${ }^{2}$, Erica K. Sloan ${ }^{6}$ and Shamgar Ben-Eliyahu ${ }^{1 *}$

Correction to: BMC Biol 18, 163 (2020)

https://doi.org/10.1186/s12915-020-00893-2

The original publication of this article [1] contained an incorrect version of figure 4, and an incorrect version of figure S4 in Additional file 1. The wrong version of both files, which contained an incorrect color legend, was accidentally used during typesetting of the article.

In this correction article the correct and incorrect version of figure 4 are published along with the updated Additional file 1.

The original article has been updated.

The original article can be found online at https://doi.org/10.1186/s12915020-00893-2.

* Correspondence: shamgar@tauex.tau.ac.il

${ }^{\dagger}$ Lee Shaashua and Anabel Eckerling contributed equally to this work.

${ }^{1}$ Sagol School of Neuroscience and School of Psychological Sciences, Tel Aviv University, 69978 Tel Aviv, Israel

Full list of author information is available at the end of the article

C The Author(s). 2020 Open Access This article is licensed under a Creative Commons Attribution 4.0 International License, which permits use, sharing, adaptation, distribution and reproduction in any medium or format, as long as you give appropriate credit to the original author(s) and the source, provide a link to the Creative Commons licence, and indicate if changes were made. The images or other third party material in this article are included in the article's Creative Commons licence, unless indicated otherwise in a credit line to the material. If material is not included in the article's Creative Commons licence and your intended use is not permitted by statutory regulation or exceeds the permitted use, you will need to obtain permission directly from the copyright holder. To view a copy of this licence, visit http://creativecommons.org/licenses/by/4.0/. The Creative Commons Public Domain Dedication waiver (http://creativecommons.org/publicdomain/zero/1.0/) applies to the data made available in this article, unless otherwise stated in a credit line to the data. 
A

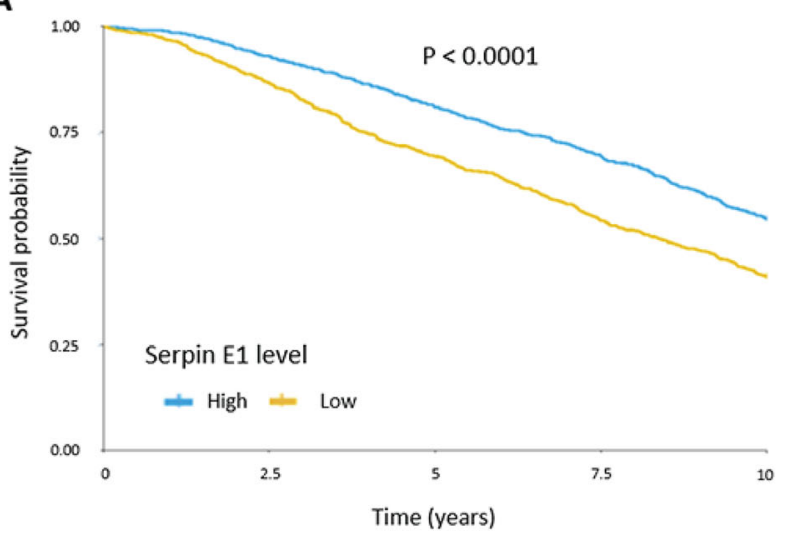

C

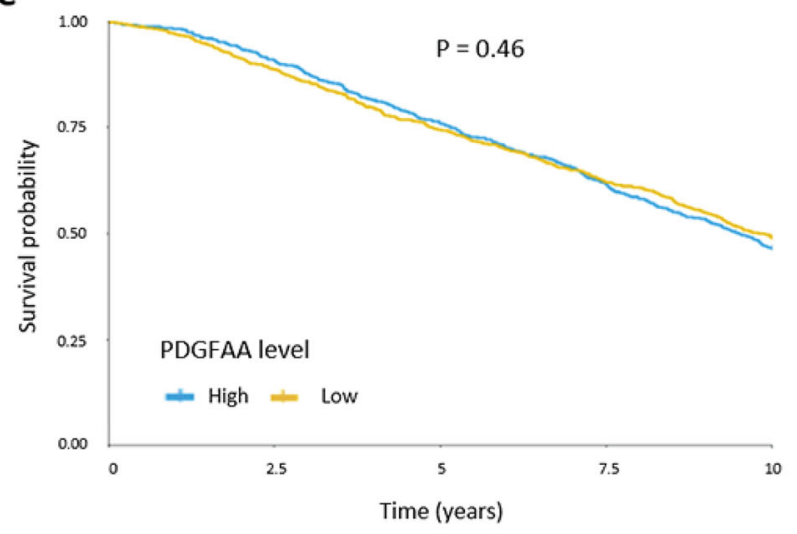

E

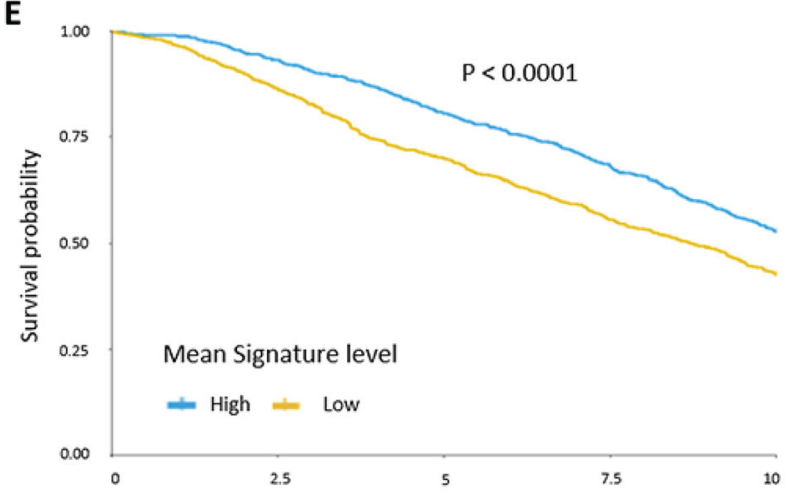

Incorrect figure 4.
B

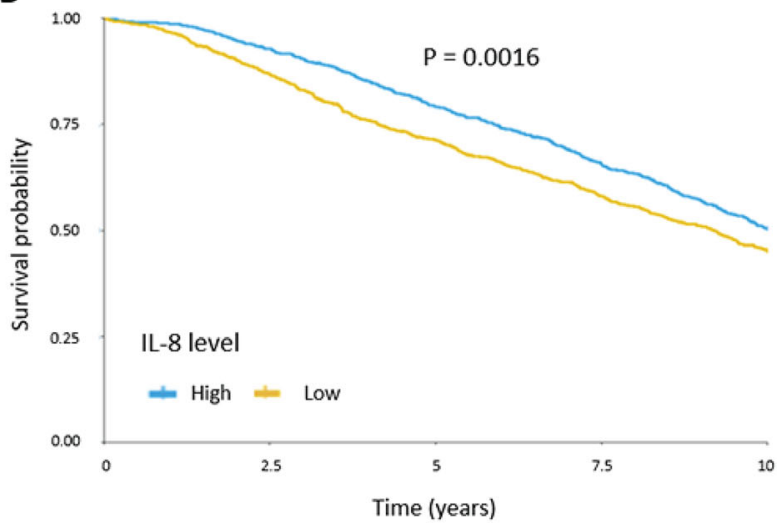

D

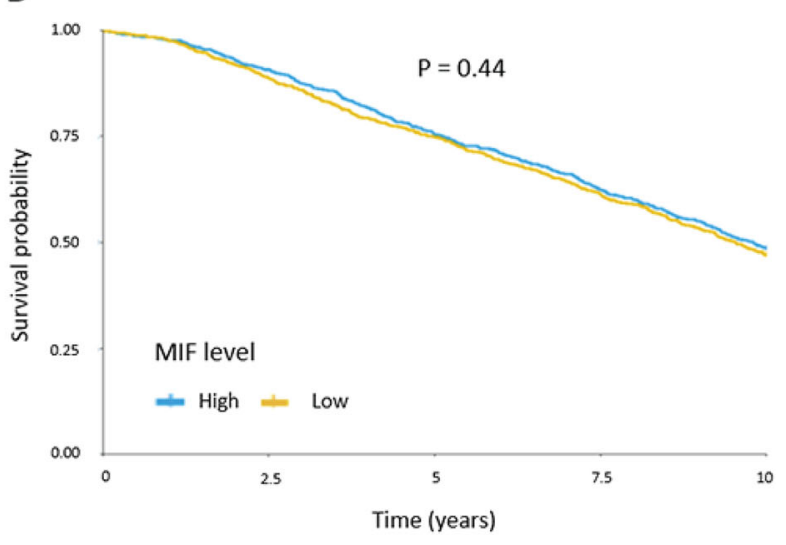




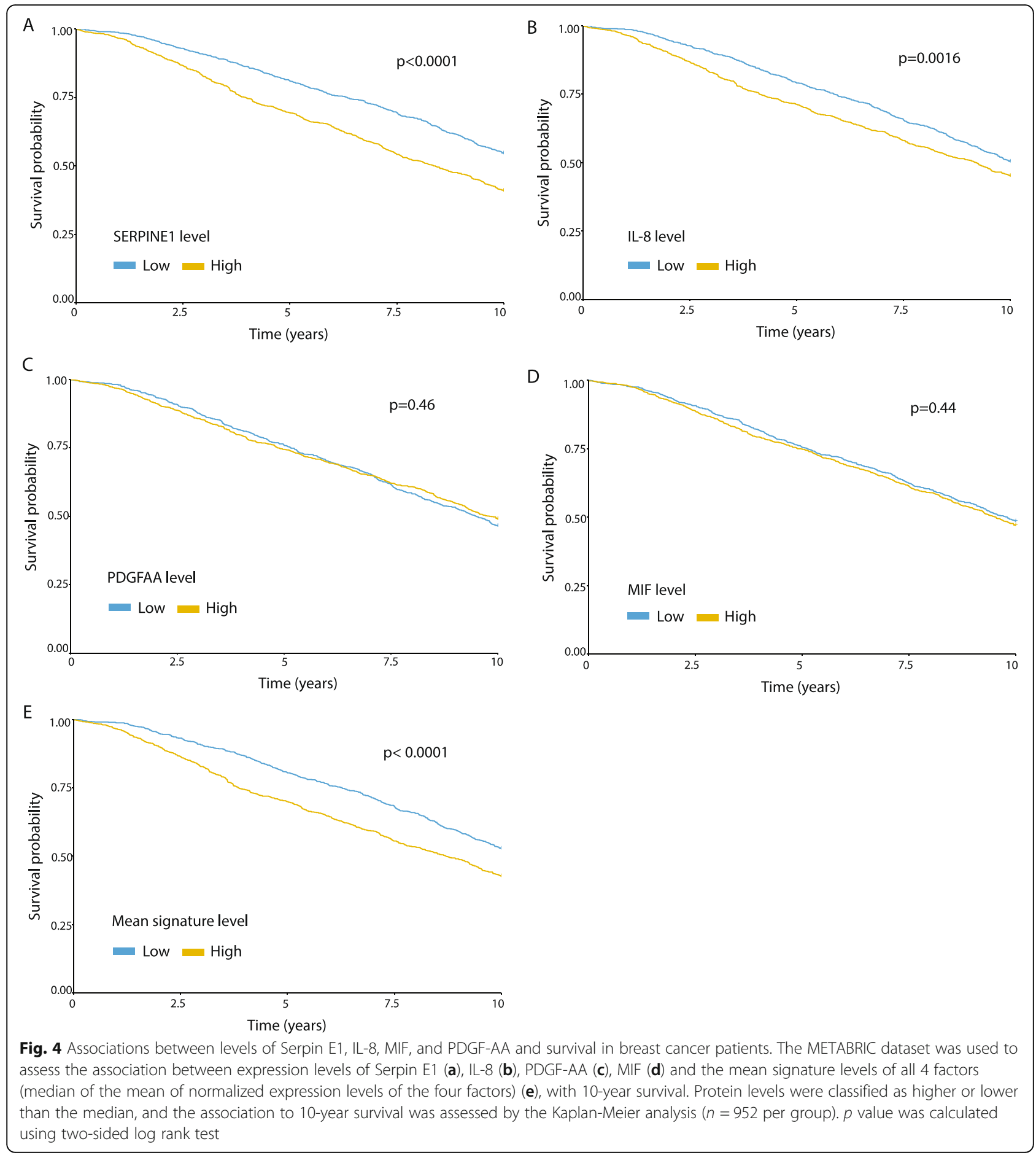

\section{Correct Figure 4.}




\section{Supplementary Information}

The online version contains supplementary material available at https://doi. org/10.1186/s12915-020-00932-y.

Additional file 1: Fig. S1. Excision of the primary tumor elicits gradual regression of early-stage metastases. Table S1. Cytokines pointed out by the cytokine array. Fig. S2. ELISA validation of in-vitro tumor secretion of the chosen cytokines. Fig. S3. Elevated levels of Serpin E1, IL-8, MIF and PDGF-AA are correlated to poor survival in lung cancer patients. Fig. S4. Associations between levels of DKK1, IL-6, M-CSF and LIF and survival in breast cancer patients.

\section{Author details}

Sagol School of Neuroscience and School of Psychological Sciences, Tel Aviv University, 69978 Tel Aviv, Israel. Department of Human Molecular Genetics and Biochemistry, Sackler Faculty of Medicine, Tel Aviv University, Tel Aviv, Israel. ${ }^{3}$ Pathology Institute, Rabin Medical Center, Tel Aviv University, Petach Tikva, Israel. ${ }^{4}$ Neurosurgery Department, Rabin Medical Center, Tel Aviv University, Petach Tikva, Israel. ${ }^{5}$ Department of Physiology and Pharmacology, Sackler Faculty of Medicine, Tel Aviv University, Tel Aviv, Israel. ${ }^{6}$ Drug Discovery Biology Theme, Monash Institute of Pharmaceutical

Sciences, Monash University, Parkville, VIC 3052, Australia.

Published online: 16 December 2020

\section{Reference}

1. Shaashua L, Eckerling A, Israeli B, et al. Spontaneous regression of micrometastases following primary tumor excision: a critical role for primary tumor secretome. BMC Biol. 2020;18:163. https://doi.org/10.1186/s12915020-00893-2. 